\title{
Methylprednisolone in adults hospitalized with COVID-19 pneumonia
}

\section{An open-label randomized trial (GLUCOCOVID)}

\author{
Luis Corral-Gudino (D) Alberto Bahamonde · Francisco Arnaiz-Revillas - Julia Gómez-Barquero · Jesica Abadía- \\ Otero - Carmen García-Ibarbia - Víctor Mora · Ana Cerezo-Hernández · José L. Hernández · Graciela López- \\ Muñíz · Fernando Hernández-Blanco · Jose M. Cifrián · Jose M. Olmos · Miguel Carrascosa · Luis Nieto · \\ María Carmen Fariñas · José A. Riancho BD · GLUCOCOVID investigators
}

Received: 9 October 2020 / Accepted: 30 December 2020 / Published online: 3 February 2021

(c) Springer-Verlag GmbH, AT part of Springer Nature 2021

The datasets generated during and/or analyzed during the current study are available from the corresponding author on reasonable request.

\section{Supplementary Information The online version of this article (https://doi.org/10.1007/s00508-020-01805-8) contains supplementary material, which is available to authorized users.}

\section{Corral-Gudino (网)}

Hospital Rio Hortega, Servicio de Medicina Interna, Universidad de Valladolid, Valladolid, Spain lcorral@saludcastillayleon.es

\section{A. Bahamonde · F. Hernández-Blanco} Servicio de Medicina Interna, Hospital Bierzo, Ponferrada, Spain

A. Bahamonde

Abahamonde@saludcastillayleon.es

F. Hernández-Blanco

Fhblanco@saludcastillayleon.es

F. Arnaiz-Revillas · M. C. Fariñas

IDIVAL, Hospital U M Valdecilla, Servicio de Enfermedades Infecciosas, Universidad de Cantabria, Santander, Spain

F. Arnaiz-Revillas

Francisco.arnaizlasrevillas@scsalud.es

M. C. Fariñas

Mcarmen.farinas@scsalud.es

J. Gómez-Barquero · J. Abadía-Otero

Hospital Rio Hortega, Servicio de Medicina Interna, Consulta de Enfermedades Infecciosas, Universidad de Valladolid, Valladolid, Spain

J. Gómez-Barquero

Gomezb@saludcastillayleon.es

J. Abadía-Otero

Jabadiao@saludcastillayleon.es
C. García-Ibarbia · J. L. Hernández · J. M. Olmos . J. A. Riancho $(\bowtie)$

IDIVAL, Hospital U M Valdecilla, Servicio de Medicina Interna, Universidad de Cantabria, Santander, Spain joseantonio.riancho@scsalud.es

C. García-Ibarbia

Carmen.garciai@scsalud.es

J. L. Hernández

Joseluis.hernandez@scsalud.es

J. M. Olmos

Josemanuel.olmos@scsalud.es

V. Mora · J. M. Cifrián

IDIVAL, Hospital U M Valdecilla, Servicio de Neumología, Universidad de Cantabria, Santander, Spain

V. Mora

Victormanuel.mora@scsalud.es

J. M. Cifrián

Josemanuel.cifrian@scsalud.es

A. Cerezo-Hernández · G. López-Muñíz

Servicio de Neumología, Hospital Universitario Río Hortega, Valladolid, Spain

\section{A. Cerezo-Hernández}

Acerezoh@saludcastillayleon.es

G. López-Muñíz

Glopezm@saludcastillayleon.es

M. Carrascosa

Servicio de Medicina Interna, Hospital Laredo, Laredo, Spain Miguel.carrascosa@scsalud.es

L. Nieto

Servicio de Medicina Interna, Hospital Sierrallana,

Torrelavega, Spain

Luishta@telefonica.net 


\section{Summary}

Purpose To determine whether a 6-day course of methylprednisolone (MP) improves outcome in patients with severe SARS-CoV-2 (Corona Virus Disease 2019 [COVID-19]).

Methods The study was a multicentric open-label trial of COVID-19 patients who were aged $\geq 18$ years, receiving oxygen without mechanical ventilation, and with evidence of systemic inflammatory response who were assigned to standard of care (SOC) or SOC plus intravenous MP ( $40 \mathrm{mg}$ bid for 3 days followed by $20 \mathrm{mg}$ bid for 3 days). The primary outcome was a composite of death, admission to the intensive care unit, or requirement for noninvasive ventilation. Both intention-to-treat (ITT) and per protocol (PP) analyses were performed.

Results A total of 91 patients were screened, and 64 were randomized (mean age $70 \pm 12$ years). In the ITT analysis, 14 of 29 patients (48\%) in the SOC group and 14 of 35 (40\%) in the MP group suffered the composite endpoint ( $40 \%$ versus $20 \%$ in patients under 72 years and $67 \%$ versus $48 \%$ in those over 72 years; $p=0.25$ ). In the PP analysis, patients on MP had a significantly lower risk of experiencing the composite endpoint (age-adjusted risk ratio 0.42; 95\% confidence interval, CI 0.20-0.89; $p=0.043$ ).

Conclusion The planned sample size was not achieved and our results should therefore be interpreted with caution. The use of MP had no significant effect on the primary endpoint in ITT analysis; however, the PP analysis showed a beneficial effect due to MP, which consistent with other published trials support the use of glucocorticoids in severe cases of COVID-19.

Keywords Glucocorticoids · SARS-CoV-2 · Mortality · Coronavirus Infections $\cdot$ Humans

\section{Introduction}

It has been generally admitted that corticosteroid therapy should not be routinely recommended in cases of viral pneumonia because the steroids might exacerbate lung injury [1]. This is the reason why guidance from the World Health Organization (WHO) at the start of the SARS-CoV-2 (COVID-19) pandemic advised against systematic use of corticosteroids in COVID-19 patients [2].

The rapid clinical deterioration of severely ill COVID-19 patients with viral pneumonia progresses into a disorder similar to the acute respiratory distress syndrome (ARDS) with multiple organ failure and death $[3,4]$. The association of increasing interleukin (IL) levels and very high acute phase reactants [5-9] act as signals for a highly systemic inflammatory response. These two findings in COVID-19 patients have led clinicians to question the recommendations against using corticosteroids. Besides, the potential benefit of corticosteroids in ARDS due to other causes has prompted interest in using them in COVID-19
[10]. During the first month of the pandemic, several authors recommended prescribing corticosteroids based on anecdotal observations and retrospective uncontrolled series of patients [11-14]. In contrast, other studies argued that corticosteroids may be deleterious and cause delayed viral clearance in COVID19 patients [15] as was also found in SARS patients [16].

On 17 July 2020, the RECOVERY trial was published [17] in which glucocorticoid use showed a clear beneficial effect in patients with COVID-19 who were on mechanical ventilation at the time of randomization in the United Kingdom (UK). Other studies have confirmed a decrease in the number of ventilator-free days (CoDEX [18]) after steroid administration. In addition, two studies of critically ill patients (CAPECOVID [19] and REMAP-CAP-COVID [20]) showed a tendency for improvement in patients receiving corticosteroids; however, both were stopped early after the RECOVERY publication. In contrast, METCOVID [21] did not show differences in mortality after a short course of methylprednisolone (MP). The evidence for the association of systemic corticosteroids with a reduction in critically ill COVID patient mortality has been summarized in two systematic reviews [22, 23].

The RECOVERY trial results showed a modest benefit of corticosteroids in less severely ill patients receiving oxygen without invasive mechanical ventilation (IMV). No benefits from the use of corticosteroids were found in patients without respiratory support on admission.

The lack of data in patients with moderate to severe disease but not yet requiring intensive care or mechanical respiratory support prompted us to report the results of a planned interim analysis of the patients who are included in our study to date. Data are needed to perform meta-analyses to better characterize the glucocorticoid outcomes in this subgroup of patients who show evidence of moderate to severe lung disease and a marked inflammatory response but still do not require mechanical ventilation.

\section{Patients, material and methods}

\section{Study design}

GLUCOCOVID is a randomized, open-label, controlled, two-arm, parallel-group, trial conducted at five hospitals in Spain (Hospital Universitario Marqués de Valdecilla, Hospital Universitario Río Hortega, Hospital El Bierzo, Hospital Laredo, and Hospital Sierrallana). The study was designed to address the efficacy of adding MP to standard therapy in patients with moderate to severe COVID-19.

We designed a pragmatic, randomized trial. In addition to the randomized trial, we also collected data from patients who had not been randomized either because the patient had a treatment preference or because their doctor preferred a specific treatment. The 
data from the patients included in this preference arm were analyzed separately from the data of the patients included in the randomized clinical trial. The reason for including this preference arm was an attempt to avoid inclusion bias in the current setting in which many physicians feel glucocorticoids may have a beneficial effect in COVID-19 despite the absence of evidence from controlled clinical trials. The incorporation of patient and/or physician preferences is based on the well-described preference trial designs, which allows incorporation of patient preferences [24, 25].

The study was registered at the European Clinical Trials Register (EudraCT number: 2020-00193437) and the Spanish Registry of Clinical Studies (2020001934-37).

\section{Participants}

Eligible patients were hospitalized subjects over 18 years of age with a laboratory confirmed diagnosis of SARS-CoV-2 infection. Additional inclusion criteria consisted of several parameters:

1. Symptom duration of at least 7 days

2. Radiological evidence of lung disease on chest X-ray or computed tomography (CT) scan

3. Moderate to severe disease with abnormal gas exchange:

(a) partial pressure of oxygen/fraction of inspired oxygen $(\mathrm{PaO} 2 / \mathrm{FiO} 2$ or $\mathrm{PaFi})<300$,

(b) arterial oxygen saturation/fraction of inspired oxygen $(\mathrm{SaO} 2 / \mathrm{FiO} 2$ or $\mathrm{SaFi})<400$ or

(c) at least two criteria of the BRESCIA-COVID Respiratory Severity Scale (BCRSS) [26].

4. Evidence of a systemic inflammatory response with any of the following: serum C-reactive protein $(\mathrm{CRP})>15 \mathrm{mg} / \mathrm{dl}$, D-dimer $>800 \mathrm{ng} / \mathrm{ml}$, ferritin $>1000 \mathrm{mg} / \mathrm{dl}$, or interleukin-6 (IL-6) levels $>20 \mathrm{pg} / \mathrm{ml}$ (the available biochemical tests in every hospital depended on local protocols).

Patients were excluded if they were mechanically ventilated, hospitalized in the intensive care unit (ICU), treated with corticosteroids or immunosuppressive drugs at the time of enrolment, had chronic kidney disease on dialysis, or were pregnant.

The study was approved by the institutional review boards of participating hospitals, and patients gave informed consent.

\section{Treatment allocation}

Once an eligible patient was identified, and the clinical team decided that a strong preference for glucocorticoid therapy existed based on either patient's and/or treating physician's preferences, the patient was allocated to the preference arm and they were not randomized. Otherwise, the patient was randomized and allocated to the MP or control arm accordingly. Patients were randomized based on a spread- sheet that transformed every medical record number into a group allocation using a concealed mathematical formula.

\section{Interventions}

Patients in both study groups received standard of care (SOC) therapy according to the local hospital protocols. The SOC protocols were similar across the participating hospitals and were based on the Spanish Ministry of Health [27] and WHO recommendations [2]. The SOC included symptomatic treatment with acetaminophen, oxygen therapy, lowmolecular weight heparin, and antibiotics for coinfections. Azithromycin, hydroxychloroquine, and lopinavir plus ritonavir were frequently prescribed.

Biochemical tests and imaging studies were performed according to clinical criteria and local protocols using standard techniques.

In addition to SOC, patients in the experimental group received intravenous (iv) MP $40 \mathrm{mg}$ bid for 3 days and then $20 \mathrm{mg}$ bid for 3 more days. The initiation of MP occurred on the same day of inclusion in the trial. The clinical teams freely prescribed interleukin-blocking agents and other therapies as indicated.

\section{Outcome}

The primary outcome measure was a composite endpoint that included in-hospital all-cause mortality, escalation to ICU admission, or progression of respiratory insufficiency that required noninvasive ventilation (NIV).

The secondary outcomes included the effects on the individual components of the composite endpoint and the variation of the laboratory biomarkers between baseline and 6 days after inclusion (time window 4-8 days).

The recorded prespecified adverse events were hyperglycemia $(>180 \mathrm{mg} / \mathrm{dl}$ based on the fasting blood glucose test) in the first 6 days after being included in the study (period of MP administration) and nosocomial infection (infection confirmed with positive cultures that was not present at the time trial inclusion but occurring within 3-28 days after first dose of MP).

\section{Sample size and statistical analysis}

With an event rate of $40 \%$ in the control arm, and a $50 \%$ risk reduction by MP, 180 patients would be needed. Due to the marked decline of new cases in our area after May 2020, the sample size could not be achieved. The study was interrupted in July 2020 after the publication of the RECOVERY trial. In this paper, we report the results of the interim analysis, which was planned a priori after inclusion of 90 patients to avoid delaying the communication of clinically useful data in the current pandemic scenario. 
Continuous variables were compared using Student's t-test and analysis of variance (ANOVA) or Mann-Whitney $U$ and Kruskal-Wallis tests if variables were not normally distributed. We compared categorical variables using Fisher's exact test. Relative risk ratio and differences in absolute risks were derived from the estimated risks of the primary composite endpoint. In stratified analyses, the combined risk ratio was computed with the Mantel-Haenszel method. Survival plots were constructed with the Kaplan-Meier method and compared with the log-rank test. Patients were followed until hospital discharge or day 28 after inclusion.

The analysis was performed according to the intention-to-treat (ITT) principle. The main analysis was complemented with a per protocol (PP) analysis. For the latter method, we considered those patients in the MP group who had received at least three doses of the drug (thus at least $24 \mathrm{~h}$ after inclusion) before the primary endpoint was reached. The treatment arms were independently studied considering the preference and randomization arms in addition to combining both MP arms.

\section{Results}

From April to July 2020, 91 patients were included in GLUCOCOVID trial (Fig. 1) and 5 patients were later eliminated from the analysis ( 2 were previously on corticosteroids, 1 was on NIV, 1 was taken to the ICU after MP initiation, and 1 patient with initial suspicion of COVID-19 was finally diagnosed with vasculitis). A total of 22 patients received MP according to the clinician's preference, and 64 patients were randomized to 1 of 2 groups ( 35 to MP and 29 to SOC). In two patients in the SOC group but none in the MP group, clinicians prescribed MP boluses after initial allocation because of deterioration of the patient's condition. One patient in SOC group received MP boluses before clinical deterioration.

Those in the MP arm were older (73 versus 66 years; $p=0.012$ ), but the baseline characteristics were otherwise very similar across groups (Table 1 and Supplementary Material, Appendix 1). In line with available protocols at the time, more than $90 \%$ of the patients received hydroxychloroquine and/or azithromycin during hospital admission. Although an active search for arrhythmia was not planned in the study protocol, no clinically significant arrhythmias were reported.

At baseline, study groups had similar biomarker levels. The CRP levels were lower in both groups 6 days later but the decrease was more pronounced in the MP group (77\% decrease versus $43 \%, p=0.034$ ). Other biomarkers were similar in the control and MP groups (Supplementary Material, Appendix 2).

In the ITT analysis, the primary composite endpoint tended to be less frequent in the MP group than in the SOC group, but the difference did not reach statistical significance with a relative risk (RR) of 0.68 (95\% confidence interval, CI $0.37-1.26 ; p=0.250)$.
Fig. 1 GLUCOCOVID flow diagram

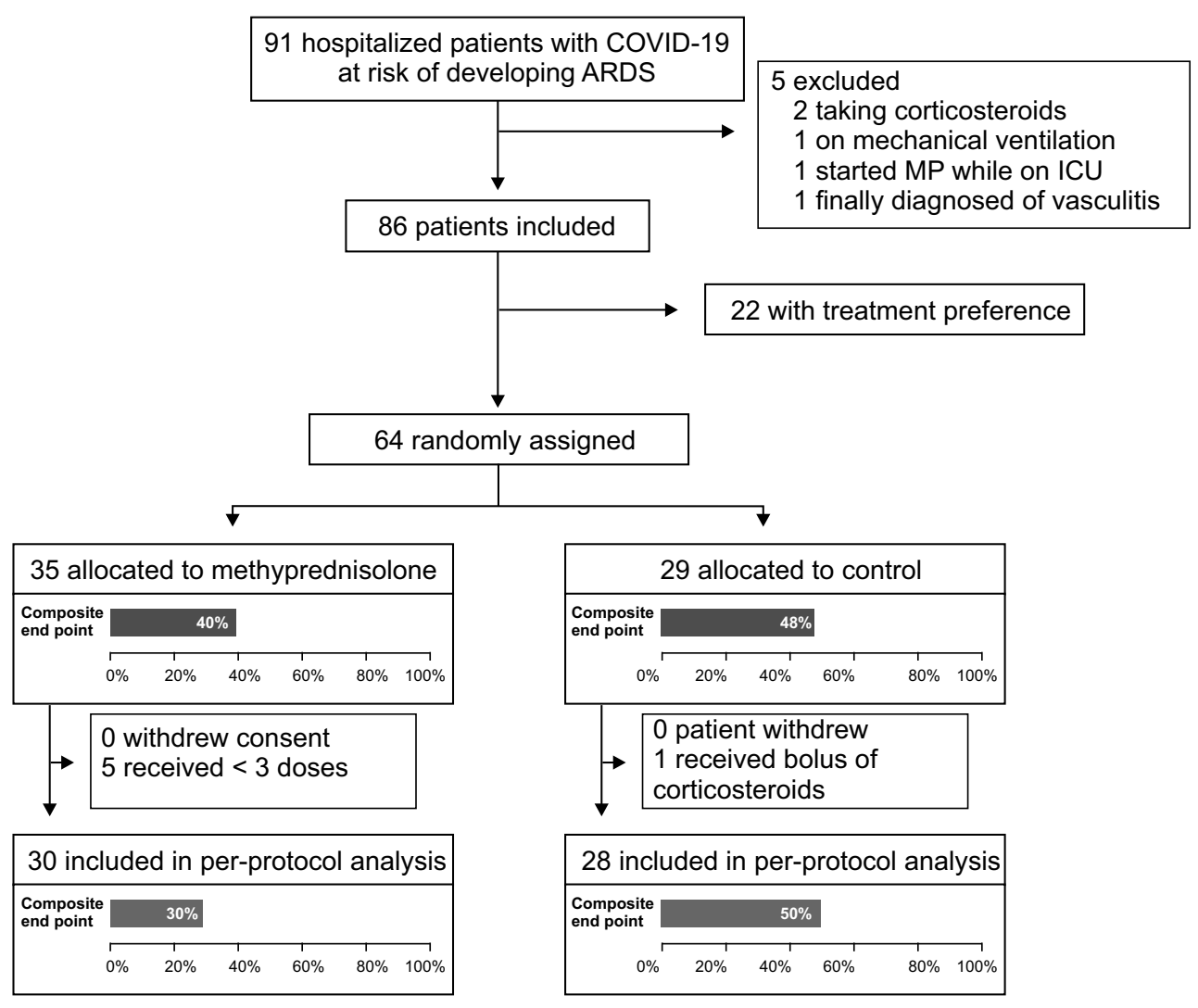


Table 1 Baseline characteristics of the study groups at randomization

\begin{tabular}{|c|c|c|c|c|}
\hline & $\begin{array}{l}\text { Total } \\
(n=64)\end{array}$ & $\begin{array}{l}\text { SOC } \\
(n=29)\end{array}$ & $\begin{array}{l}\mathrm{MP} \\
(n=35)\end{array}$ & $\begin{array}{l}\text { Mean differences SOC vs. MP } \\
(95 \% \mathrm{Cl})\end{array}$ \\
\hline Age, years, mean $\pm S D$ & $70 \pm 12$ & $66 \pm 12$ & $73 \pm 11$ & $-7(-13$ to -2$)$ \\
\hline Sex (male, \%) & $39(61)$ & $16(55)$ & $23(66)$ & $-11 \%(-33$ to 13$)$ \\
\hline Days from symptom onset to inclusion, mean \pm SD & $12 \pm 6$ & $12 \pm 7$ & $12 \pm 5$ & 0.3 (-2.6 to 3.2$)$ \\
\hline \multicolumn{5}{|l|}{ COVID-19 characteristics } \\
\hline SAFI (Sa02/FI 02), median (IQR) & $262(179-350)$ & $319(169-406)$ & $254(180-337)$ & 18 (-38 to 74$)$ \\
\hline Creatinine, mg/dl, median (IQR) & $0.9(0.7-1.1)$ & $0.9(0.7-1.1)$ & $0.9(0.8-1.1)$ & $-0.1(-0.3$ to 0.2$)$ \\
\hline Lymphocytes $10^{9} / L$, median (IQR) & $0.8(0.6-10)$ & $0.8(0.6-10)$ & $0.8(0.6-10)$ & $-0.2(-0.6$ to 0.3$)$ \\
\hline Platelets $10^{9} / \mathrm{L}$, median (IQR) & $232(180-335)$ & $244(215-359)$ & $216(159-338)$ & $19(-41$ to 80$)$ \\
\hline CRP, mg/dl, median (IQR) & $16(12-24)$ & $16(11-24)$ & $16(12-24)$ & $0.1(-4$ to 4$)$ \\
\hline D-dimer, ng/ml, median (IQR) & $1240(681-2093)$ & $980(557-1856)$ & $1340(712-2152)$ & $-1912(-4879$ to 1054$)$ \\
\hline Ferritin, mg/dl, median (IQR) & $1052(517-1504)$ & $976(449-1355)$ & $1100(627-1574)$ & $-182(-661$ to 297$)$ \\
\hline qSOFA (points), median (IQR) & $1(1-1)$ & $1(1-1)$ & $1(1-1)$ & $-0.06(-0.3$ to 0.2$)$ \\
\hline \multicolumn{5}{|l|}{ Comorbidities } \\
\hline Hypertension, $n(\%)$ & $30(47)$ & $12(41)$ & $18(51)$ & $-10 \%(-32$ to 14$)$ \\
\hline Cardiac disease, $n(\%)$ & $8(13)$ & $4(14)$ & $4(11)$ & $3 \%$ (-14 to 20$)$ \\
\hline Respiratory disease, $n(\%)$ & $5(8)$ & $1(3)$ & $4(11)$ & $-8 \%(-22$ to 7$)$ \\
\hline Diabetes, $n(\%)$ & $11(17)$ & $4(14)$ & $7(20)$ & $-6 \%(-24$ to 13$)$ \\
\hline \multicolumn{5}{|l|}{ Therapy } \\
\hline Azithromycin, $n(\%)$ & $58(91)$ & $29(100)$ & $29(83)$ & $17 \%$ (-2 to 33$)$ \\
\hline Hydroxychloroquine, $n(\%)$ & $61(95)$ & $29(100)$ & $32(91)$ & $9 \%(-4$ to 22$)$ \\
\hline Lopinavir/ritonavir, $n(\%)$ & $53(83)$ & $28(97)$ & $25(71)$ & $26 \%$ (7 to 42 ) \\
\hline $\begin{array}{l}\text { LMWH (prophylactic dose), } n(\%) \\
\text { LMWH (anticoagulant dose), } n(\%)\end{array}$ & $\begin{array}{l}49(77) \\
9(14)\end{array}$ & $\begin{array}{l}22(76) \\
4(14)\end{array}$ & $\begin{array}{l}27(77) \\
5(14)\end{array}$ & $\begin{array}{l}1 \%(-22 \text { to } 18) \\
0.5(-17 \text { to } 18)\end{array}$ \\
\hline
\end{tabular}

Table 2 Comparison of patients in the control and methylprednisolone (MP) arms. Age-stratified analyses

\begin{tabular}{|c|c|c|c|c|}
\hline Outcome & Methylprednisolone & Standard of care & Relative risk $(95 \% \mathrm{Cl})$ & $p$ \\
\hline \multicolumn{5}{|c|}{ Intention-to-treat } \\
\hline$<72$ years & $2 / 10(20 \%)$ & $8 / 20(40 \%)$ & $0.50(0.13-1.93)$ & 0.273 \\
\hline$\geq 72$ years & $12 / 25(48 \%)$ & $6 / 9(67 \%)$ & $0.72(0.39-1.33)$ & 0.336 \\
\hline Total & $14 / 35(40 \%)$ & $14 / 29(48 \%)$ & $0.68(0.37-1.26)^{\mathrm{a}}$ & 0.250 \\
\hline \multicolumn{5}{|l|}{ Per protocol } \\
\hline$<72$ years & $1 / 9(11 \%)$ & $8 / 20(40 \%)$ & $0.28(0.04-1.90)$ & 0.120 \\
\hline$\geq 72$ years & $8 / 21(38 \%)$ & $6 / 8(75 \%)$ & $0.51(0.26-1.00)$ & 0.074 \\
\hline Total & $9 / 30(30 \%)$ & $14 / 28(50 \%)$ & $0.42(0.20-0.89)^{\mathrm{a}}$ & 0.043 \\
\hline
\end{tabular}

Advanced age was unbalanced between group and significantly associated with an adverse outcome $(p=0.024)$. Thus, to adjust age imbalance between groups, we performed an age-adjusted analysis by stratifying patients into two groups. We selected the median age (72 years) as the cut-off to split the groups (Table 2 and Fig. 2). Neither the average time from symptom onset to inclusion $(11 \pm 6$ days for the patients who achieved the endpoint, $12 \pm 5$ days for patients who did not achieve the endpoint, difference -1 day, $95 \%$ CI $-4-2$ days) nor the average time from hospital admission to inclusion ( $4 \pm 6$ days versus $4 \pm 4$ days, difference -0.3 days, $95 \%$ CI $-2-3$ days) were associated with the achievement of the endpoint. Data of the individual components of the primary endpoint are described in detail in Sect. 3 of Supplementary Material.

In the PP analysis, we included patients who received at least three MP doses before the composite endpoint occurred (this step indicates at least $24 \mathrm{~h}$ elapsed between inclusion and the occurrence of an endpoint event). The use of MP was associated with a significantly lower risk of the composite outcome with a $20 \%$ absolute risk reduction ( $30 \%$ versus $50 \%)$ and RR 0.42 (95\% CI $0.20-0.89 ; p=0.043$ ) as shown in Table 2 and Supplementary Material, Appendix 3. In the age-stratified analysis, RR was 0.28 (95\% CI $0.04-1.90$ ) in the group up to 72 years of age and 0.51 
Randomized patient $(\mathrm{n}=64)$ [intention to treat]

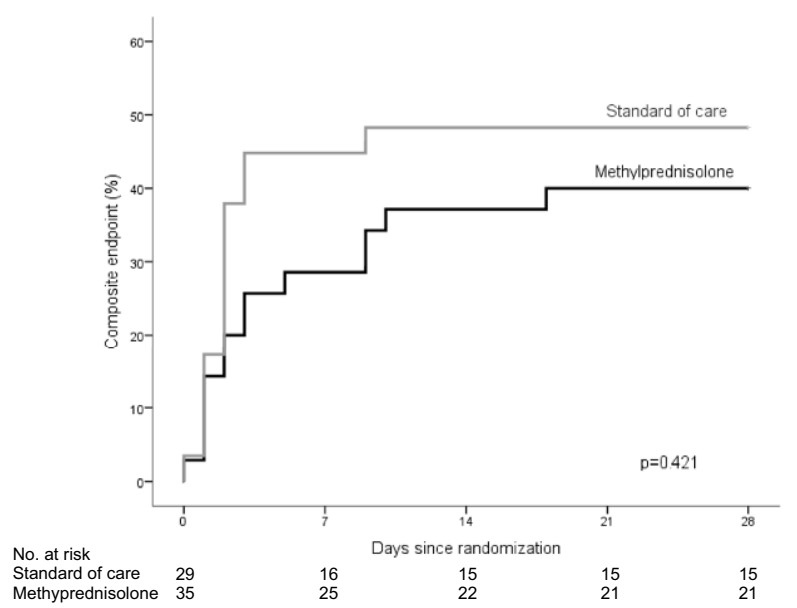

Randomized patient according age group $(\mathrm{n}=64)$ [intention to treat]

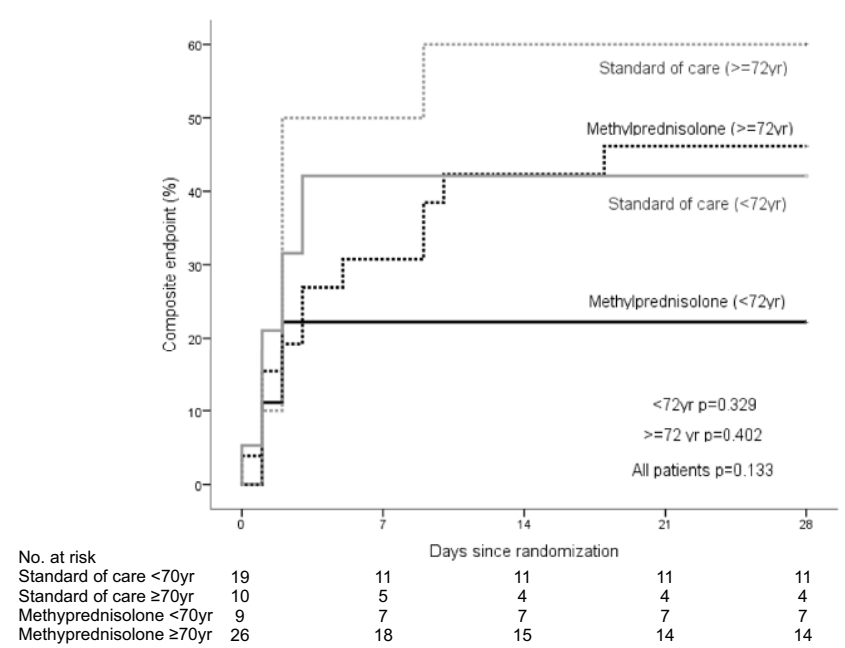

a b

Fig. 2 Intention to treat analysis. Kaplan-Meier plots showing the probability of occurrence of the primary composite endpoint (ICU admission, need of NIV or death) on the control vs. methylprednisolone groups (a Total group, $\mathbf{b}$ stratified by age)

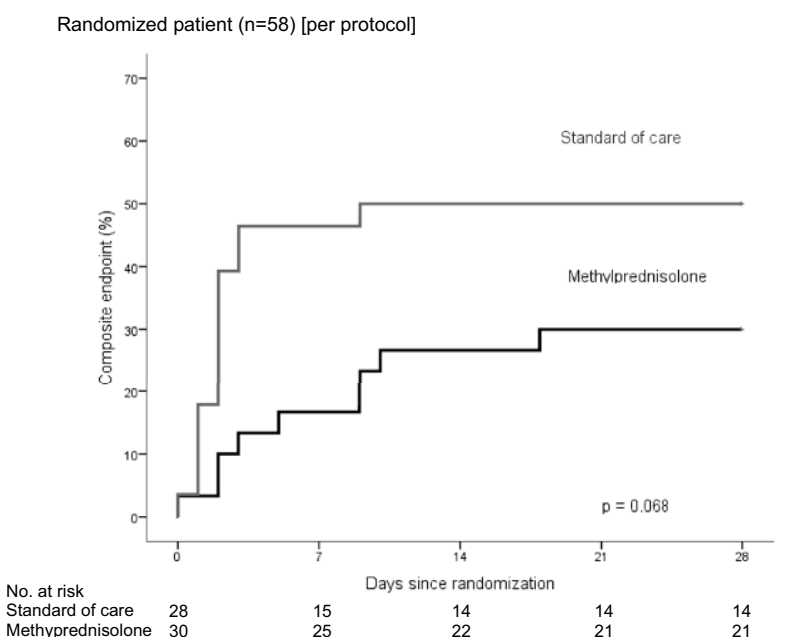

a
Randomized patient according age group $(n=58)$ [per protocol]

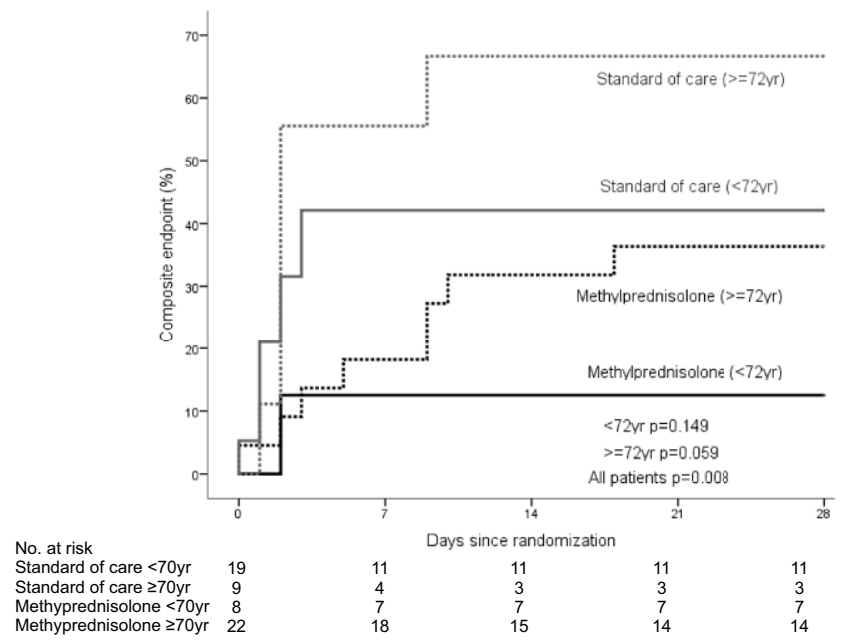

Fig. 3 Per protocol analysis. Kaplan-Meier plots showing the probability of occurrence of the primary composite endpoint (ICU admission, need of NIV or death) on the control vs. methylprednisolone groups (a Total group, $\mathbf{b}$ stratified by age)

(95\% CI $0.26-1.00)$ in the group over 72 years (Table 2). Thus, although age was a confounding variable, no statistically significant interaction between age and the effect of MP was noted. Survival analysis confirmed those results with a significant difference between SOC and MP groups ( $p=0.008$, Fig. 3 ).

Of the patients 10 received the IL- 6 blocking agent, tocilizumab, $6(17 \%)$ in the MP randomized group and $4(14 \%)$ in the control group; in four patients on the MP group (11\%), the IL-1 blocking agent, anakinra, was prescribed. After patients treated with tocilizumab and/or anakinra were excluded from the analysis, the results were similar to those found in the whole group, RR 0.62 (95\% CI 0.31-1.22) in intention to treat analysis, and RR 0.48 (95\% CI $0.22-1.05)$ in per protocol analysis.

Patients receiving MP in the preference arm had similar characteristics to those in the randomized groups (Supplementary Material, Appendix 1). The comparison of the whole MP group (combining patients in both MP arms, preference or randomized) and the SOC group confirmed the beneficial effect of MP both in the intention to treat analysis (ageadjusted RR 0.56, 95\% CI 0.32-0.96, $p=0.041$ ) and in the per protocol analysis (age adjusted RR 0.33, $95 \%$ CI $0.17-0.65, p=0.003$ ) (supplementary material, appendix 3).

Hyperglycemia $(>180 \mathrm{mg} / \mathrm{dl})$ was more frequent in the MP group (9 patients in the MP group, $26 \%$, and 
none in the SOC group, $p=0.015)$. Nosocomial infections also tended to be more common in the MP group ( $14 \%$ versus $3 \% p=0.637$ ) as shown in Supplementary Material and Appendix 4. The length of stay in the hospital was similar for both groups (SOC $20 \pm 14$ days, MP $23 \pm 13$ days, difference 3 days, 95\% CI -10-4 days).

\section{Discussion}

In this trial, MP administration was associated with a reduced risk of primary outcome as shown in the PP analysis but not in the ITT analysis. Due to the decline of new cases in our area after the implementation of social distancing and quarantining in March 2020 and the premature end of our trial after the publication of RECOVERY [17] results, we were not able to achieve the planned sample size. In the PP analysis, we included patients who received at least three MP doses; however, we did not include those who experienced an endpoint event less than $24 \mathrm{~h}$ after the first MP dose in the analysis. Albeit somewhat arbitrary, we reasoned that $24 \mathrm{~h}$ was the shortest time to experience a drug beneficial effect. Furthermore, similar results were obtained in the analysis combining the preference and randomized arms.

Despite the limited statistical power of our sample, the outcomes of our PP analysis are consistent with already published data. The RECOVERY trial showed a lower 28-day mortality in patients receiving a 10day course of dexamethasone among hospitalized patients with severe COVID-19 (patients were receiving either oxygen alone or IMV). The METCOVID trial confirmed this beneficial effect with a short course of MP only in patients over 60 years with severe COVID 19 (with $\mathrm{SpO} 2 \leq 94 \%$ in room air, use of supplementary oxygen, or under IMV) [21]. Our study did not include critically ill patients or patients with mild disease. Nevertheless, the study results confirm the beneficial effects of glucocorticoids in a well-defined subset of patients, including those with evidence of abnormal gas exchange and systemic inflammatory response, who are at risk of developing ARDS, thus becoming critically ill and requiring IMV; however, close follow-up is needed, paying particular attention to glucocorticoid-related side effects, such as hyperglycemia and higher risk of nosocomial infections.

Patients in the MP arm were somewhat older than those in the control arm. This finding is an important issue in this study since we confirmed that advanced age is a risk factor for poor outcome in this study, a finding that is in line with previously reported series $[12,28,29]$. The confounding effect of age is complex as it may influence not only the course of the disease but also decisions about escalation to ICU admission in a scenario of limited resources. Thus, age stratification is important for an adequate interpretation of the results. In the METCOVID trial [21] the authors observed only a reduction in mortality with MP use in patients over 60 years old but not in younger individ- uals. We did not find evidence of interaction between treatment and patient age. In other words, MP had a similar beneficial effect both in younger and older individuals in our study as opposed to the findings from the METCOVID trial in which the youngest patients seem to have more fatal outcomes. The sample size of our study hampered the possibility for achieving significance in individual endpoints, but there was a change in the direction of the effects with more fatal outcomes in the SOC group of patients who were $\geq 72$ years $(50 \%$ in SOC group versus $24 \%$ in the MP group, difference $26 \%$, 95\% CI -9-58\%, in PP analysis). Our results are also consistent with those of a recent quasi experimental study [14] that used similar endpoints and MP doses. The primary composite endpoint occurred in $54 \%$ patients in the SOC group and in $35 \%$ in the early glucocorticoid group. Those figures resemble our PP analysis (50\% versus $30 \%$ ). On the negative side, hyperglycemia and coinfections, especially in the ICU, appear to be the more frequent adverse events in the MP group.

Our study has several limitations. First, the sample size was not achieved due to the aforementioned reasons. Although statistical significance was achieved in PP analysis and in the ITT analysis by combining the randomized and preference arms, our study is underpowered to detect treatment differences in the ITT analysis limited to the randomized arms, particularly for subgroup analyses and the secondary outcomes; however, we feel that the results are important enough to guide clinical decisions and to better define the effects described by larger controlled randomized trials [30]. Second, the inclusion of a preferential arm theoretically hampers the balance of baseline characteristics across study arms. Thus, we restricted the main analysis to the randomized arms. Nevertheless, the analysis combining all patients who received MP confirmed the main results. Third, differences in patient management across the participating hospitals might have existed. Fortunately, in practice the protocols for COVID-19 were remarkably similar because they were based on the recommendations of the Spanish Ministry of Health that included the use of azithromycin, hydroxychloroquine, and lopinavir/ ritonavir. Fourth, due to the rapidly deteriorating course of some COVID-19 patients, they were transferred to the ICU or NIV within the first $24 \mathrm{~h}$ of study inclusion. Therefore, they received only 1 or 2 doses of MP, thus impeding assessment of the effects of the drug. We included a PP analysis excluding those patients in an attempt to obtain a measure of drug effect that would be more relevant from the practical point of view; however, since such analysis departs from the more robust ITT analysis, the results should be interpreted with caution.

In summary, although the study was terminated before achieving the complete sample size and no significant differences in ITT analysis were found, our PP analysis suggests that MP most likely has a clin- 
ically relevant effect in reducing the risk of developing severe respiratory insufficiency and ARDS, thus helping clinicians tailor therapy in non-mechanically ventilated patients with lung disease and systemic inflammation. These data should be interpreted with caution along with the outcomes of other trials of corticosteroid use in COVID-19 patients.

Acknowledgements We thank Professor Jose Luis PérezCastrillón (Universidad de Valladolid) for useful discussion and comments on the manuscript, and TICNOR (Santander, Spain) for providing the database mobile application. This trial would have been impossible without the support and collaboration of the hundreds of health professionals (clinicians, microbiologists, radiologists, biochemists, nurses, technologists, administrative personnel) involved in the care of COVID19 patients in our hospitals during the 2020 spring pandemic.

Author Contribution Substantial contributions to conception and design: J.A. Riancho, M.C. Fariñas, J.M. Cifrián, J.M. Olmos, M. Carrascosa, and F. Arnaiz-Revillas. Acquisition of data: L. Corral-Gudino, A. Bahamonde, F. ArnaizRevillas, J. Gómez-Barquero, J. Abadía-Otero, C. García-Ibarbia, V. Mora, A. Cerezo-Hernández, J.L. Hernández, G. LópezMuñíz, F. Hernández-Blanco, J.M. Cifrián, J.M. Olmos, M. Carrascosa, L. Nieto, M.C. Fariñas, and J.A. Riancho. Analysis and interpretation of data: L. Corral-Gudino and J.A. Riancho. Drafting the article or revising critically for important intellectual content: L. Corral-Gudino, A. Bahamonde, F. Arnaiz-Revillas, J. Gómez-Barquero, J. Abadía-Otero, C. García-Ibarbia, V. Mora, A. Cerezo-Hernández, J.L. Hernández, G. López-Muñíz, F. Hernández-Blanco, J.M. Cifrián, J.M. Olmos, M. Carrascosa, L. Nieto, M.C. Fariñas, and J.A. Riancho. Final revision of the version to be published: L. Corral-Gudino, A. Bahamonde, F. Arnaiz-Revillas, J. Gómez-Barquero, J. Abadía-Otero, C. García-Ibarbia, V. Mora, A. CerezoHernández, J.L. Hernández, G. López-Muñíz, F. HernándezBlanco, J.M. Cifrián, J.M. Olmos, M. Carrascosa, L. Nieto, M.C. Fariñas, and J.A. Riancho.

\section{Compliance with ethical guidelines}

Conflict of interest L. Corral-Gudino, A. Bahamonde, F. Arnaiz-Revillas, J. Gómez-Barquero, J. Abadía-Otero, C. García-Ibarbia, V. Mora, A. Cerezo-Hernández, J.L. Hernández, G. López-Muñíz, F. Hernández-Blanco, J.M. Cifrián, J.M. Olmos, M. Carrascosa, L. Nieto, M.C. Fariñas, J.A. Riancho, and GLUCOCOVID investigators declare that they have no competing interests.

Ethical standards All human and animal studies were approved by the hospital ethics committee and therefore were performed in accordance with the ethical standards laid down in the 1964 Declaration of Helsinki and its later amendments. All procedures followed were in accordance with the ethical standards of the responsible committee on human experimentation (institutional and national) and with the Helsinki Declaration of 1975 , as revised in 2008. The study was registered at the European Clinical Trials Register (https:/ / eudract. ema.europa.eu/ EudraCT number: 2020-001934-37) and the Spanish Registry of Clinical Studies (https://reec.aemps.es/ reec/public/web.html. Number 2020-001934-37). Informed consent was obtained from all patients included in the study.

\section{Appendix}

\section{Glucocovid investigators}

\section{Hospital El Bierzo}

Alberto Bahamonde, Fernando Hernández-Blanco, Cristina Buelta-González, Luis A. Marcos-Martínez, Ana I. Martínez-Vidal, Pilar R.l Dosantos-Gallego, Jesús Pérez-Sagredo, Silvia Sandomingo-Freire, Rebeca Muñumer-Blázquez, Antonio Paredes-Mogollo, Elena Brague-Allegue.

\section{Hospital Laredo}

Miguel Carrascosa, Juan L. García-Rivero.

\section{Hospital Sierrallana}

Luis Nieto.

\section{Hospital Universitario Marqués de Valdecilla}

José A. Riancho, José M. Olmos, Carmen Fariñas, José M. Cifrian, Carmen García-Ibarbia, Jose L. Hernández, Francisco Arnaiz-Revillas, Victor Mora, Sara Nieto, Juan Ruiz-Cubillán, Arancha Bermúdez, Javier Pardo, Carlos Amado, Andrés Insunza, Aritz Gil, Teresa Diaz-Terán, Marina Fayos, Miguel A. Zabaleta, Juan J. Parra.

\section{Hospital Unviersitario Río Hortega}

Luis Corral-Gudino, Julia Gómez-Barquero, Jesica Abadía-Otero, Ana Cerezo-Hernández, Graciela LópezMuñíz, Angela Ruíz-de-Temiño-de-la-Peña, C. Ainhoa Arroyo-Domingo, Javier Mena-Martín, José Pablo Miramontes-González, Ana E Jiménez-Masa, Luis Pastor-Mancisidor, Tanía M Álvaro-de-Castro, María Cruz Pérez-Panizo, Tomás Ruíz-Albi, C Gema de-la-ColinaRojo, María Andrés-Calvo, Andrea Crespo-Sedano, Begoña Morejón-Huerta, Laisa S. Briongos-Figuero, Julio F Frutos-Arriba, Javier Pagán-Buzo, Miriam Gabella-Martín, Marta Cobos-Siles, Ana Gómez-García.

\section{References}

1. Russell CD, Millar JE, Baillie JK. Clinical evidence does not support corticosteroid treatment for 2019-nCoV lung injury. Lancet. 2020;395:473-5.

2. Clinical management of severe acute respiratory infection when COVID-19 is suspected [.. https://www.who. int/publications-detail/clinical-management- of-severeacute-respiratory-infection-when-novel-coronavirusncov-infection-is-suspected. Accessed 2 Apr 2020.

3. Guan W-J, Ni Z-Y, Hu Y, Liang W-H, Ou C-Q, He J-X, et al. Clinical Characteristics of Coronavirus Disease 2019 in China. NEngl J Med. 2020;382:1708-20.

4. Cevik M, Bamford CGG, Ho A. COVID-19 pandemic-a focused review for clinicians. Clin Microbiol Infect. 2020;26:842-47. https://doi.org/10.1016/j.cmi.2020.04. 023.

5. Mehta P, McAuley DF, Brown M, Sanchez E, Tattersall RS, Manson JJ, et al. COVID-19: consider cytokine 
storm syndromes and immunosuppression. Lancet. 2020;395:1033-4.

6. Siddiqi H, Mehra M. COVID-19 illness in native and Immunosuppresed states: a clinical-therapeutic staging proposal. JHeart Lung Transplant. 2020;39:405-7.

7. McGonagle D, Sharif K, O'Regan A, Bridgewood C. The role of cytokines including Interleukin-6 in COVID-19 induced pneumonia and macrophage activation syndromelike disease. Autoimmun Rev. 2020;19:102537.

8. Zhou F, Yu T, Du R, Fan G, Liu Y, Liu Z, et al. Clinical course and risk factors for mortality of adult inpatients with COVID-19 in Wuhan, China: a retrospective cohort study. Lancet. 2020;395:1054-62.

9. Cobos-Siles M, Cubero-Morais P, Arroyo-Jiménez I, ReyHernández M, Hernández-Gómez L, Vargas-Parra DJ, et al. Cause-specific death in hospitalized individuals infected with SARS-CoV-2: more than just acute respiratory failure or thromboembolic events. Intern Emerg Med. 2020;15:1533-44. https://doi.org/10.1007/s11739020-02485-y.

10. VillarJ,FerrandoC, MartínezD, AmbrósA, MuñozT, SolerJA, et al. Dexamethasone treatment for the acute respiratory distress syndrome: a multicentre, randomised controlled trial. LancetRespir Med. 2020;8:267-76.

11. Zhou W, Liu Y, Tian D, Wang C, Wang S, Cheng J, et al. Potential benefits of precise corticosteroids therapy for severe 2019-nCoV pneumonia. Signal Transduct Target Ther. 2020;5:18.

12. Wu C, Chen X, Cai Y, Xia J, Zhou X, Xu S, et al. Risk factors associated with acute respiratory distress syndrome and deathin patientswith Coronavirus disease2019pneumonia in Wuhan, China. JAMA Intern Med. 2020;180:934-43. https://doi.org/10.1001/jamainternmed.2020.0994.

13. Zha L, Li S, Pan L, Tefsen B, Li Y, French N, et al. Corticosteroid treatment of patients with coronavirus disease 2019 (COVID-19). Med J Aust. 2020;212:416-20.

14. Fadel R, Morrison AR, Vahia A, Smith ZR, Chaudhry Z, Bhargava P, et al. Early short course corticosteroids in hospitalized patients with COVID-19. Clin Infect Dis. 2020;71:2114-20. https://doi.org/10.1093/cid/ciaa601.

15. Ling Y, Xu S-B, Lin Y-X, Tian D, Zhu Z-Q, Dai F-H, et al. Persistence and clearance of viral RNA in 2019 novel coronavirus disease rehabilitation patients. Chin Med J. 2020;133:1039-43.

16. Stockman LJ, Bellamy R, Garner P. SARS: systematic review of treatment effects. PLoS Med. 2006;3:e343.

17. RECOVERY Collaborative Group, Horby P, Lim WS, Emberson JR, Mafham M, Bell JL, et al. Dexamethasone in hospitalized patients with Covid-19-preliminary report. NEngl JMed. 2020. https://doi.org/10.1056/NEJMoa2021436.

18. Tomazini BM, Maia IS, Cavalcanti AB, Berwanger O, Rosa RG, Veiga VC, et al. Effect of dexamethasone on days alive and ventilator-free in patients with moderate or severe acute respiratory distress syndrome and COVID-19: the coDEX randomized clinical trial. JAMA. 2020;324:1307-16. https://doi.org/10.1001/jama.2020.17021.

19. Dequin P-F, Heming N, Meziani F, Plantefève G, Voiriot G, Badié J, et al. Effect of hydrocortisone on 21-day mortality or respiratory support among critically ill pa- tients with COVID-19: a randomized clinical trial. JAMA. 2020;324:1298-1306. https://doi.org/10.1001/jama.2020. 16761 .

20. Writing Committee for the REMAP-CAP Investigators, Angus DC, Derde L, Al-Beidh F, Annane D, Arabi Y, et al. Effect of hydrocortisone on mortality and organ support in patients with severe COVID-19: the REMAP-CAP COVID-19 corticosteroid domain randomized clinical trial. JAMA. 2020;324:1317-29. https://doi.org/10.1001/jama. 2020.17022.

21. Prado-Jeronimo CM, Farias MEL, Val FFA, Sampaio VS, Alexandre MAA, Melo GC, et al. Methylprednisolone as adjunctive therapy for patients hospitalized with COVID19 (Metcovid): a randomised, double-blind, phase IIb, placebo-controlled trial. Clin Infect Dis. 2020. https://doi. org/10.1093/cid/ciaal177.

22. WHO Rapid Evidence Appraisal for COVID-19 Therapies (REACT) Working Group, Sterne JAC, Murthy S, Diaz JV, Slutsky AS, Villar J, et al. Association between administration of systemic corticosteroids and mortality among critically ill patients with COVID-19: a meta-analysis. JAMA. 2020;324:1330-41. https://doi.org/10.1001/jama. 2020.17023.

23. Siemieniuk RA, Bartoszko JJ, Ge L, Zeraatkar D, Izcovich A, KumE, et al. Drug treatments for covid-19: living systematic review and network meta-analysis. BMJ. 2020;370:m2980.

24. Wasmann KA, Wijsman P, van Dieren S, Bemelman W, Buskens C. Partially randomised patient preference trials as an alternative design to randomised controlled trials: systematic review and meta-analyses. BMJ Open. 2019;9:e31151.

25. Kowalski CJ. When ethics precludes randomization: put prospective, matched-pair observational studies to work. Perspect Biol Med. 2013;56:184-97.

26. MDCalc. Brescia-COVID Respiratory Severity Scale (BCRSS)/Algorithm.. https://www.mdcalc.com/bresciacovid-respiratory-severity-scale-bcrss-algorithm. Accessed 13Apr 2020.

27. Agencia Española deMedicamentosy Productos Sanitarios. Tratamientos disponibles para el manejo de la infección respiratoria por SARS-CoV-2.. https://www.aemps.gob. es/la-aemps/ultima-informacion-de-la-aemps-acercadel-covid\%e $2 \% 80 \% 9119 /$ tratamientos-disponibles-parael-manejo-de-la-infeccion-respiratoria-por-sars-cov- $2 /$. Accessed 15 Apr 2020.

28. Jordan RE, Adab P, Cheng KK. Covid-19: risk factors for severe disease and death. BMJ. 2020;368:m1198.

29. Grasselli G, Zangrillo A, Zanella A, Antonelli M, Cabrini L, Castelli A, et al. Baseline characteristics and outcomes of 1591 patients infected with SARS-coV-2 admitted to ICus of the Lombardy region, Italy. JAMA. 2020;323:1574-81.

30. Halpern SD, Karlawish JHT, Berlin JA. The continuing unethical conduct of underpowered clinical trials. JAMA. 2002;288:358-62.

Publisher's Note Springer Nature remains neutral with regard to jurisdictional claims in published maps and institutional affiliations. 